\title{
Hepatosplenic Cat Scratch Disease: Description of Two Cases Undergoing Contrast-Enhanced Ultrasound for Diagnosis and Follow-Up and Systematic Literature Review
}

\author{
Daniela Tirotta $^{1} \cdot$ Vincenzo Mazzeo $^{1} \cdot$ Maurizio Nizzoli $^{1}$
}

Accepted: 26 April 2021 / Published online: 15 June 2021

(C) The Author(s), under exclusive licence to Springer Nature Switzerland AG 2021

\begin{abstract}
Cat scratch disease (CSD) is a disease usually characterized by self-limited lymphadenopathy of the young man. Rarely CSD, however, can manifest itself as an unusual hepatosplenic form (HS-CSD) in immunocompetent patients. HS-CSD diagnosis is generally based on clinical features, imaging, and serologies, but sensitivity of serologies is very variable, like that of other diagnostic methods, as Warthin-Starry silver stain and isthology. Also there are no specific markers for the follow-up. The use of the CEUS (abdominal contrast-enhanced ultrasound) in HS-CSD is not previously described in literature examined, but we think that CEUS can be of help to diagnosis and follow-up of these patients, even after an initial CT scan, because it is a sensitive method, as seen in other diseases associated with granulomas, such as sarcoidosis. We describe 2 new cases of HS-CSD, and we performed a systematic review of the clinical cases reported in the past 10 years in the literature associated to an analysis of clinical, diagnostic, and therapeutic aspects of the disease.
\end{abstract}

Keywords Cat scratch disease $\cdot$ Immunocompetent patient $\cdot$ Hepatosplenic cat scratch disease $\cdot$ Abdominal contrast-enhanced ultrasound $\cdot$ Diagnosis $\cdot$ Follow-up

\section{Introduction}

Cat scratch disease (CSD) is a disease transmitted from a scratch or bite of an infected cat (rarely dog), as well as from cat flea or contact with cat saliva through broken skin or mucosal surfaces. CSD is usually characterized by self-limited lymphadenopathy of the young man. In these patients, the use of antimicrobials is rarely necessary $[1,2]$.

Rarely CSD, however, can include visceral, neurologic, and ocular involvement (in 5-20\% of patients), and, in particular, an unusual hepatosplenic form (HS-CSD) is described in $2.3 \%$ of patients.

Bartonella henselae (B. henselae) can cause intraerythrocytic bacteremia of the cat, for 1 year or longer [3]. In humans, generally $B$. henselae causes a local infection, but it can also invade

This article is part of the Topical Collection on Medicine

Daniela Tirotta

danitirotta@libero.it

1 Morgagni-Pierantoni Hospital, Forli’ (AUSL Romagna), Internal Medicine Unit, Via Carlo Forlanini, 47121, Forlì, Italy endothelial cells, and it can cause an acute inflammatory reaction associated to activation of a proinflammatory cascade [4]. Why some patients have a local self-limited lymphadenopathy and others a disseminated disease (sometimes associated to lifethreatening complications) is unclear.

\section{Patients and Methods}

We present 2 cases of HS-CSD observed in immunocompetent patients and a subsequent systematic review of CSD cases published in the literature in the last 10 years. We performed a systematic research on PubMed for cases of Bartonella henselae infection with liver and spleen involvement (Mesh term "Cat Scratch Disease" AND "liver" AND/OR "spleen", with detailed control of references). We have extended the search to web search engine (Google), using the following keywords: "Cat Scratch Disease," "hepatic and spleen involvement," "Bartonella henselae", "immunocompetent adults," and "Cat scratch disease."

We obtained 42 cases; we excluded 27 for temporal limits (temporal limits: 2010-2020), age limit (adult: $19+$ years), and language limit (English language) with total of 15 cases (Table 1). 


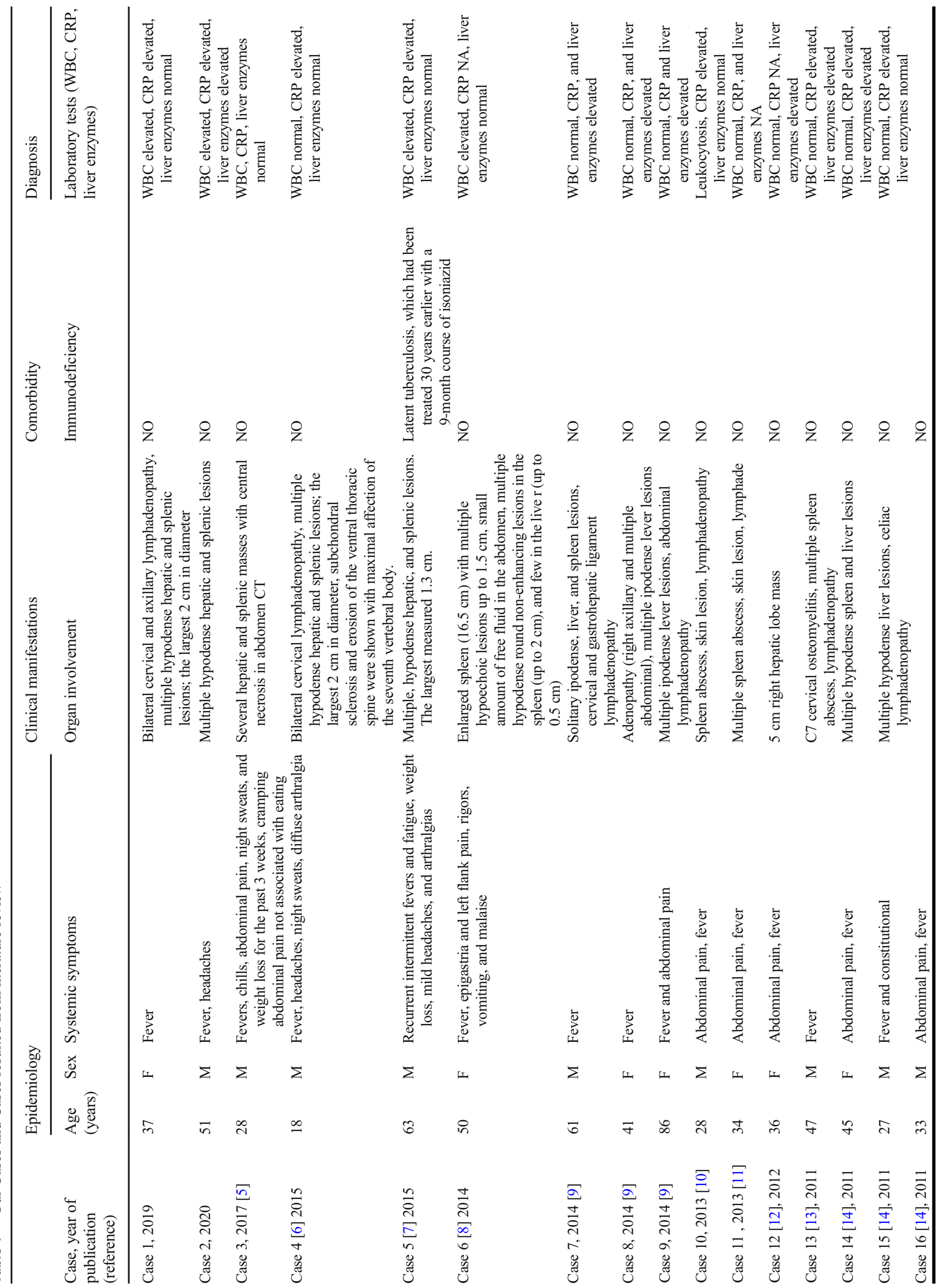




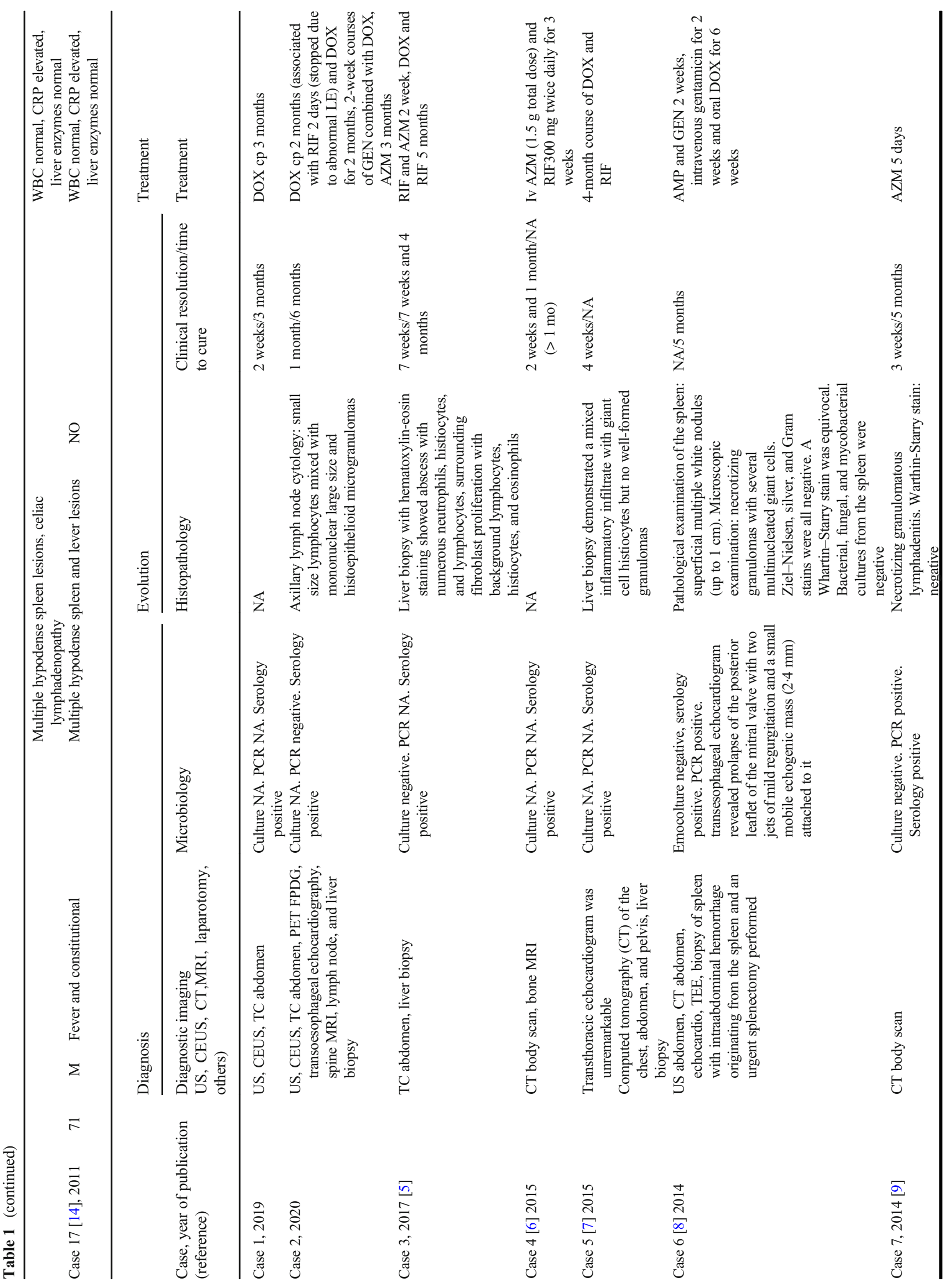




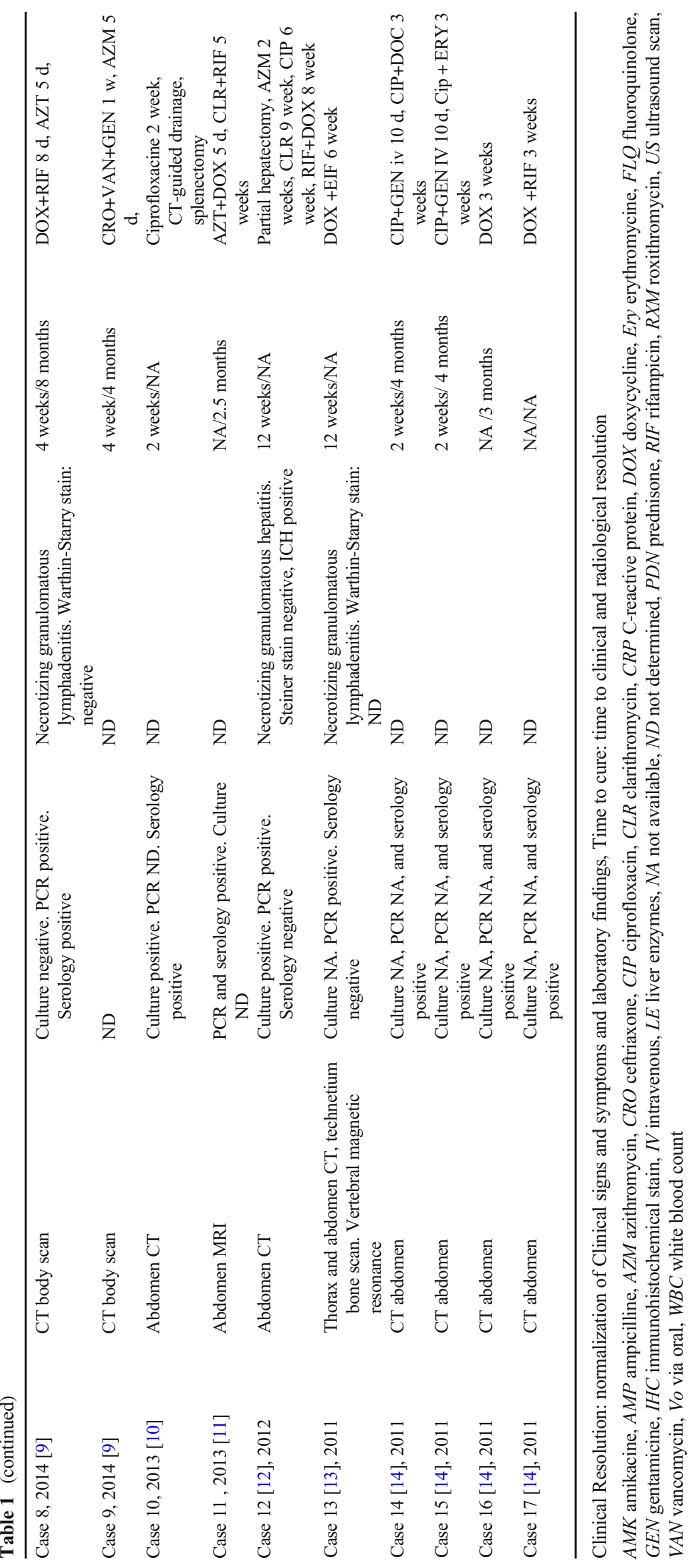


Demographic data (sex, age), epidemiologic data, clinical presentation, possible immunodeppression status, laboratory findings, radiologic image, surgery and histologic findings, clinical course, and treatments were reported and discussed.

\section{Results}

\section{Case Series}

\section{Case 1}

A 33-year-old woman was admitted in 2019 in the Internal Medicine Unit due to continuous fever for 1 week and splenomegaly.

Her medical history was negative. She denied alcohol or drug use. She did not report cat scratch or bite in the weeks before admission, but she had a cat.

Our abdomen ultrasound (US) showed multiple hypoechoic splenic lesions and splenomegaly. Chestabdomen CT confirmed multiple nodular lesions compatible with splenic abscess. CEUS (contrast-enhanced ultrasound), after administration of Sonovue $1 \mathrm{f} \mathrm{ev}$, showed several nodular splenic lesions (the max diameter was $1.5 \mathrm{~cm}$ ) without arterial wash-in and without venous wash-out (Fig. 1).

Laboratory data revealed neutrophilic leukocytosis (WBC $8000 / \mathrm{mmc}$ ) and biological inflammation syndrome (CRP 100 $\mathrm{mg} / \mathrm{l} \gg 10 \mathrm{mg} / \mathrm{l})$; liver and kidney tests were normal. Bartonella IgM was positive, repeated at 5 days (IgM 100, IgG positive, IFA test), and blood cultures and electrophoresis were negatives. We performed also serology for Toxoplasma, Borrelia, Cytomegalovirus, and Epstein-Barr that resulted negative. Defervescence occurred after 1 week of antibiotic therapy (doxycycline). The patient was discharged with doxycycline $100 \mathrm{mg} 1 \mathrm{cp} \times 2$. After 3 months, the lesions disappeared on abdomen US and CEUS, which we used for follow-up of patient.

\section{Case 2}

A 51-year-old man with no past medical history presented to our Internal Medicine Unit in 2020 with fever (up to 39 C for 6 days), headache, and superficial lymphadenomegaly.

He reported a cat scratch in the right hand in the weeks before admission, but no bite. He denied alcohol or drug use.

Laboratory studies showed neutrophilic leukocytosis (WBC 10400> 6000/mmc) and biological inflammation syndrome (CRP $116>>21 \mathrm{mg} / \mathrm{l}$ ), and liver and kidney tests and electrophoresis were normal.

We performed in our Internal Medical Unit an abdomen US (which showed splenomegaly with multiple hypoechoic lesions of maximum $14 \mathrm{~mm}$ ) and a CEUS: after administration of Sonovue $1 \mathrm{f} \mathrm{ev}$, we did not observed in the arterial phase wash-in of the described focal lesions of the spleen and the liver (1.56 cm maximum diameter), except weakly in the periphery of the nodular lesions of the spleen, and we did not observe wash-out in the venous phase (Fig. 2.1, 2.2 and 2.3).

An immunofluorescence assay (IFA) detected the presence of IgM antibodies against $B$. henselae with a titer of $\operatorname{IgG}<64$. After 5 days, titer of igM was 128 , and $\operatorname{IgG}$ was positive. Blood cultures were negative.

We performed also two COVID-19 swabs and serology for Toxoplasma, Borrelia, and Mycoplasma that resulted negative and Legionella and Pneumococcus antigens and quantiferon that resulted negative.

The following instrumental tests were performed:

- Chest CT: some lymphadenomegalies in the right axillary area, with a reactive appearance (maximum diameter $27 \times 14 \mathrm{~mm})$.
Fig. 1 CEUS of patient 1. Spleen, arterial phase: nodular splenic lesions without arterial wash-in and without venous wash-out

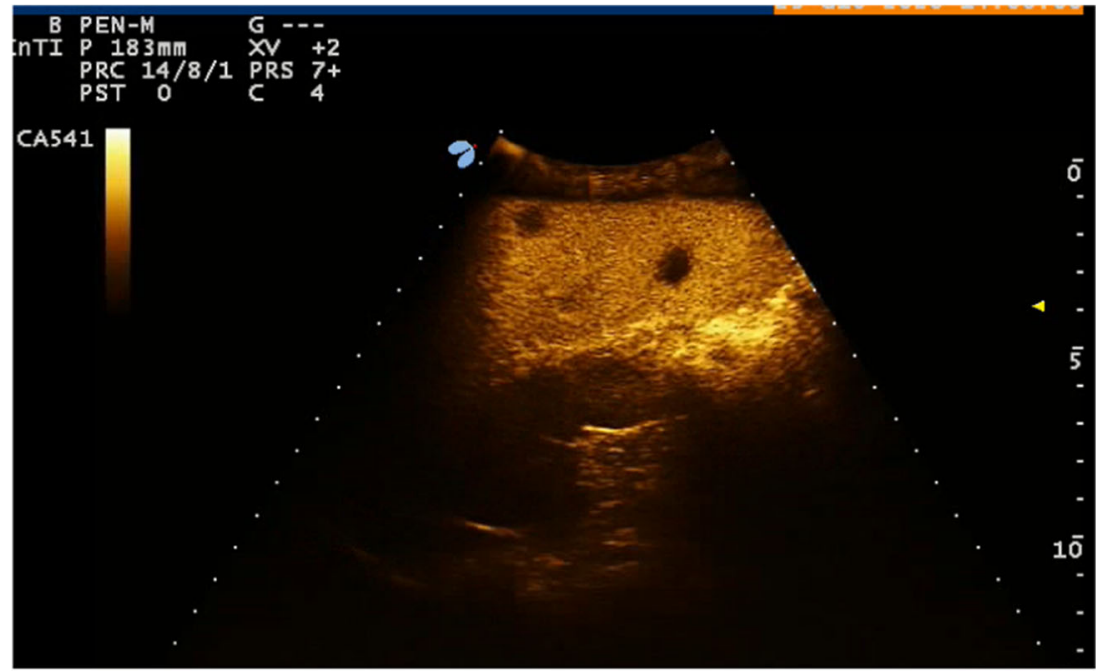




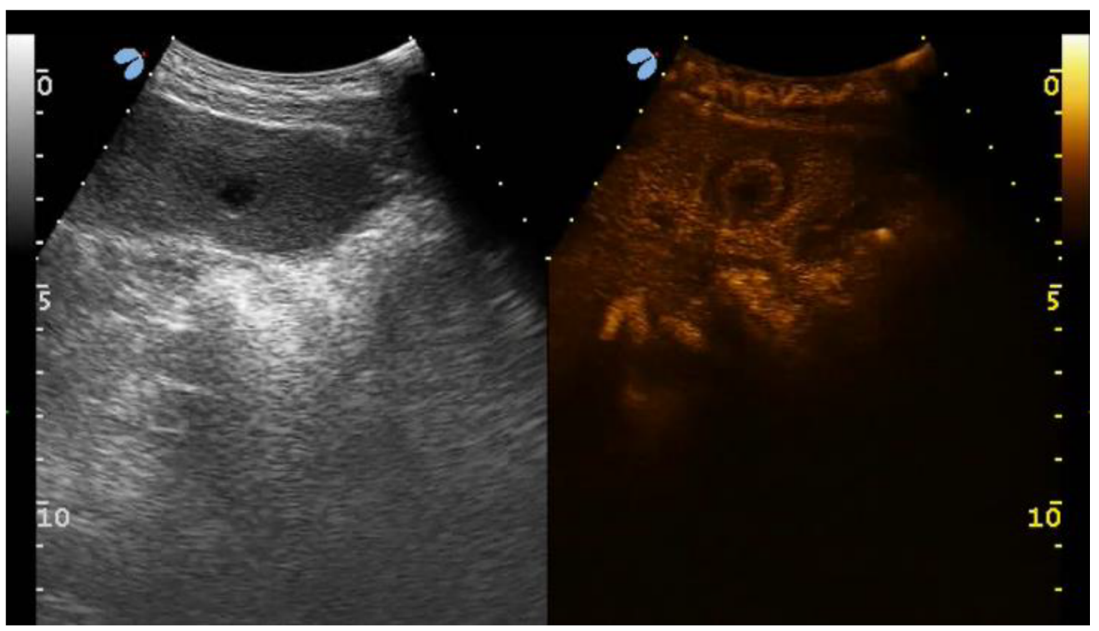

Fig. 2.1 CEUS patient 2. Left liver, arterial phase: nodular liver lesions with peripheral arterial wash-in and without venous wash-out

- Abdomen CT: hepatomegaly associated to some subcentimetric and hypodense nodular lesions, some of which after administration of contrast agent showed peripheral rim enhancement (microabscesses? Not characterized because of small size); splenomegaly (diameter $15 \mathrm{~cm}$ ) with hypodense nodular formations (greater than $10 \mathrm{~mm}$ ), some of which associated to peripheral rim enhancement, periaortic and interaortocaval lymphadenomegalies (max diameter $17 \mathrm{~mm}$ ), and lymphadenopathy in hepatic hilum (28 x15 mm) (Fig. 3).

- Axillary lymph node cytology: small size lymphocytes mixed with mononuclear large size and histoepithelioid microgranulomas (Fig. 4).

- Brain CT: negative.

- Lumbar puncture for CSF analysis (performed for neck rigidity and headache): clear liquid, meningitis was excluded through chemical and physical examination.

- $\quad$ 18F-FDG PET scans: accumulation of agent contrast in the axillary lymph node (SUV 13.44), lymph nodes of the hepatic hilum, some hepatic and splenic inhomogeneity, vertebral D11 hyperaccumulation.

- Transparietal echocardium: thickening of the noncoronary aortic cusp, non-specific, associated to mild regurgitation.

- Transesophageal echocardium: no suspicious images for vegetations (performed 4 days later).

- Eye examination: no uveitis.

- Spine MRI: inhomogeneity in the posteroinferior edge of the soma of D11.
We prescribed antibiotic therapy with doxycycline and rifampicin, but because of the onset of a cholestatic hepatitis, then we administered only doxycycline.

The patient became apyretic and asymptomatic after 10 days.

$\mathrm{He}$ was discharged with diagnosis of Bartonella endocarditis in the early phase (3 minor criteria of Dukes and one major) in course of HS-CSD, complicated by bone localization of the posteroinferior edge of D11 and intercurrent iatrogenic cholestatic liver disease.

One month later, he performed abdomen US, which showed at least five hypoechoic images of the liver (maximum diameter $1.8 \mathrm{~cm}$ ) and of the spleen (maximum diameter 1.7 $\mathrm{cm})$. CEUS, used for follow-up, showed a nodular lesion not previously present in the VII segment (Fig. 2.3).

Laboratory tests showed decreased CRP $(8.2 \mathrm{~mm} / \mathrm{l})$ and normal liver tests.

Recent medical history was positive for photosensitivity reaction in the hands and occasional lower back pain.

The patient was hospitalized again. Blood tests showed Bartonella seroconversion (negative IGM, IgG 512, IFA test), procalcitonin, complement, lymphocyte typing, blood cultures were normal.

We repeated echocardium (negative), lumbosacral MRI: minimal L1 inhomogeneity, and PET positive for persistent hepatosplenic uptake with no accumulation of the contrast in L1 and SUV attenuation in abdominal lymph nodes.

He performed a double liver biopsy: we sent a sample to S Matteo in Pavia for PCR Bartonella test (negative) and one to the Anatomy Pathology of Forli ' (liver parenchyma was normal). 
Fig. 2.2 One month later. CEUS of patient. Liver and spleen, arterial phase. New nodular lesion without arterial wash-in and without venous wash-out in VII segment
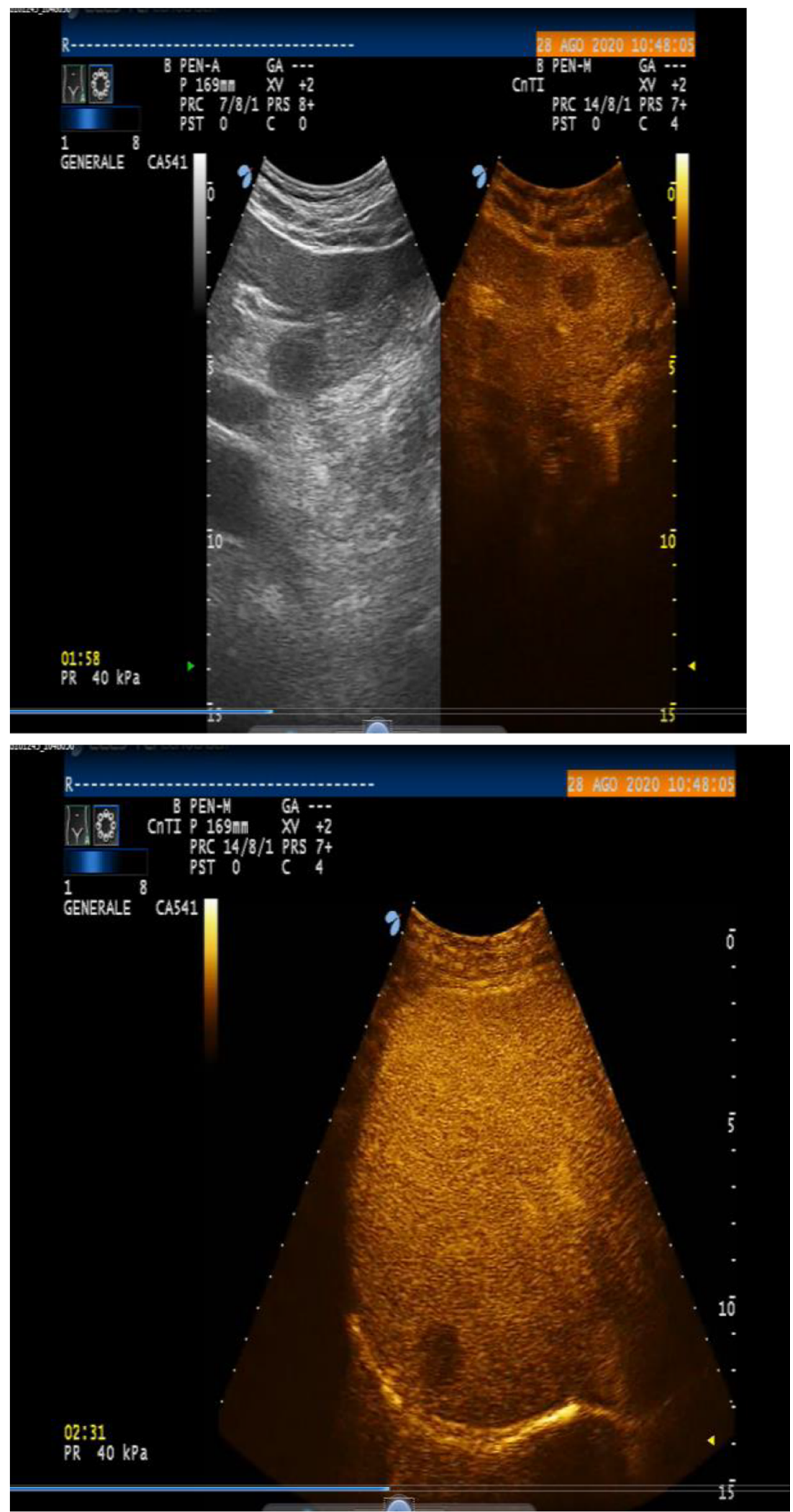
Fig. 2.3 CEUS of patient, six months later. Liver and spleen, arterial phase. Resolution of nodular lesion
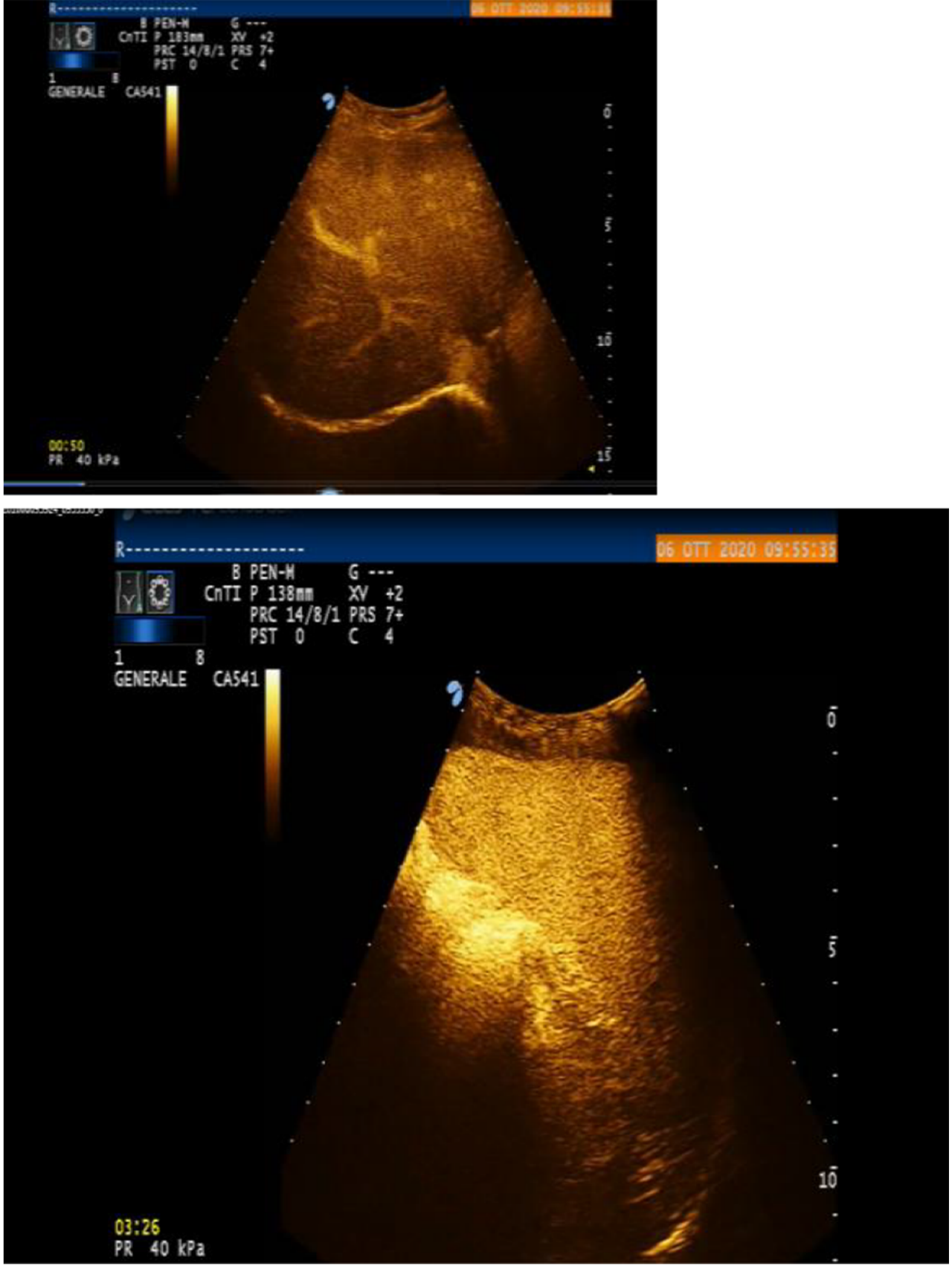

After the biopsy, a new therapeutic scheme began: gentamicin in single administration $(3 \mathrm{mg} / \mathrm{kg})$ and azithromycin intravenous 2 weeks and then azithromycin orally.

Six months later, abdomen US combined with CEUS showed resolution of liver and splenic lesions (Fig. 2.3).

Table 1 summarizes the characteristics of the other $15 \mathrm{pa}-$ tients described in the literature.

\section{Discussion}

Our case series and cases of literature review stress how HS CSD has multisystem involvement and how there is a lack of definitive diagnostic tests, so diagnosis and management of HS-CSD is often difficult.

Epidemiology CSD is commonly a disease of children and young adults, but a surveillance study in Israel showed that $6 \%$ of immunocompetent patients with CSD was $\geq 60$ years old [15]. The mean age of patients observed is 44.47 years and median 41 age (range $28-86$ years). In total $7 / 17 \%$ of patients are women.

Atipical CSD In the literature, older CSD patients have more frequently atypical locations (Table 2 showed major manifestation of atypical SCD): in a surveillance study in 
Fig. 3 Abdomen CT of patient 2.

Liver and splenic lesions

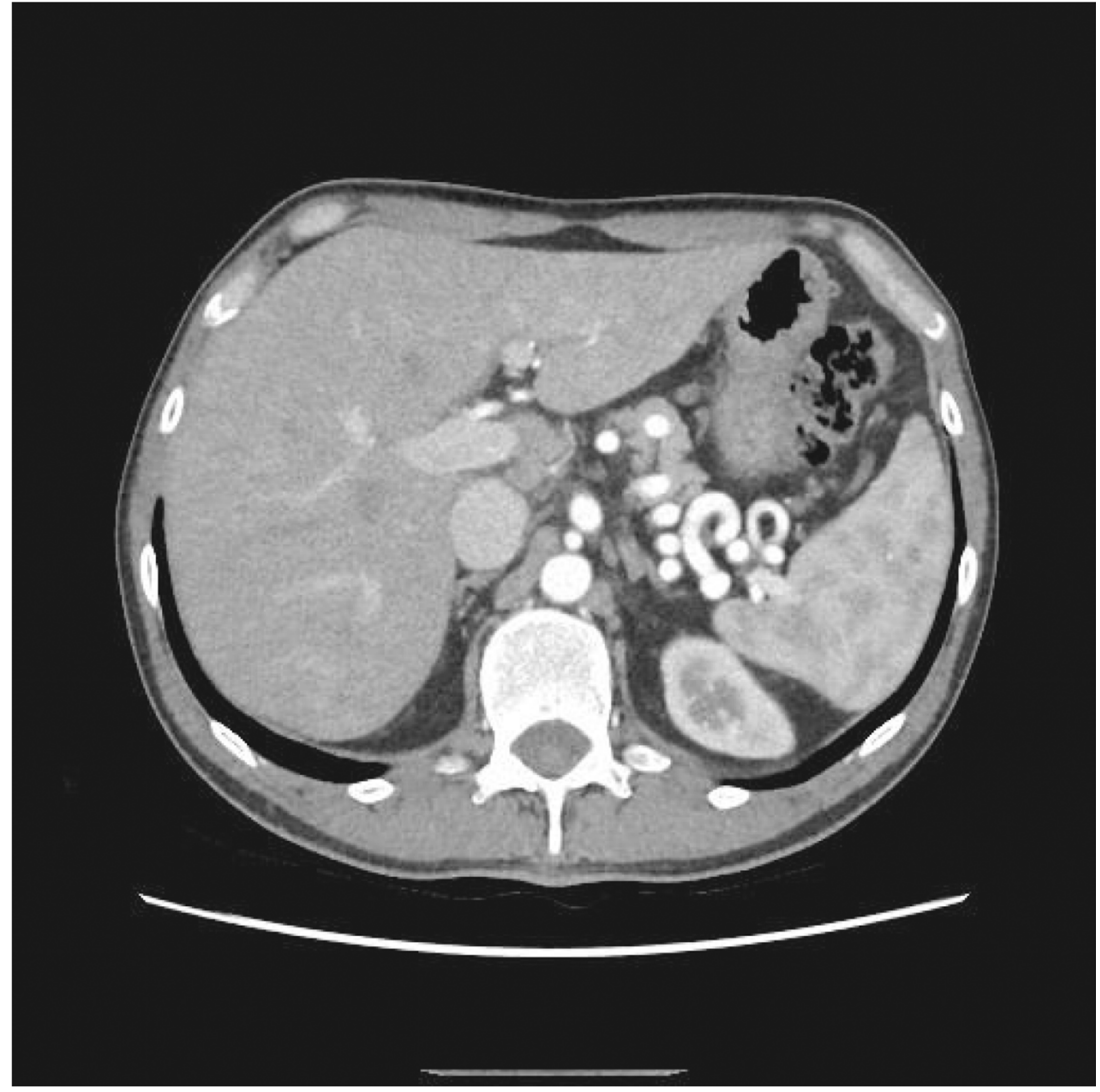

Israel, lymphadenitis (typical of CSD) was less common in the elderly (aged $\geq 60$ years) ( 77 versus $94 \%$ ), while the elderly have more likely general malaise ( 71 versus 51 percent) and atypical manifestation. For this reason, the diagnostic latency is higher in elderly (commonly $>6$ weeks). In our case series and review, patients with HS-CSD are 4/17 (23.5\%), and elderly patients ( $>60$ years) are not the majority of cases.
Hepatosplenic localitation in cat scratch disease is very rare, and in literature, it is often described in children [16-18]. In literature only $10 \%$ of patients with $B$. henselae infection develop hepatic granuloma or splenic abscess. Similarly, bone involvement, which we observed in patient 2 , is rare (only $0.27 \%$ develop osteomyelitis and bone localization, most often in the vertebral column). In our systematic
Fig. 4 Lymphnode cytology: small size lymphocytes mixed with mononuclear large size and histoepithelioid microgranulomas

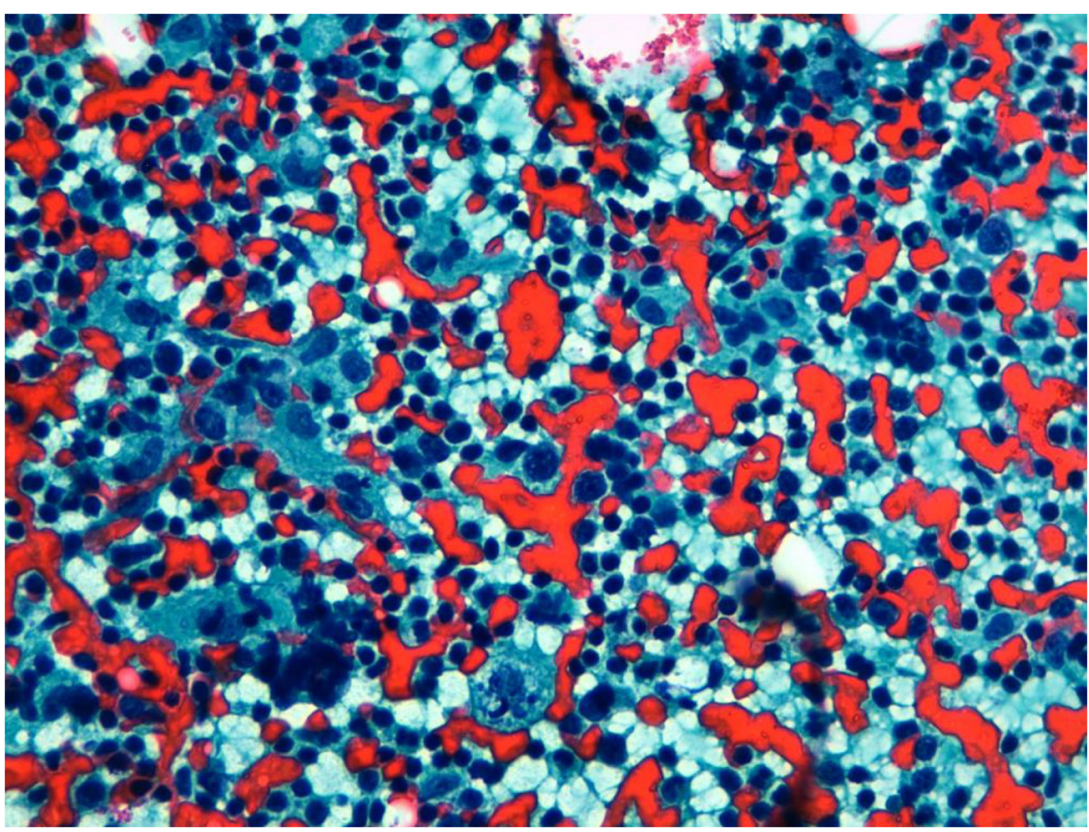


Table 2 Typical and atypical CSD manifestations

\begin{tabular}{ll} 
Immunocompromised patient & Immunocompetent patient \\
\hline $\begin{array}{l}\text { 1. Bacillary angiomatosis (cutaneous lesions resemble } \\
\text { Kaposi's sarcoma) }\end{array}$ & $\begin{array}{c}\text { Typical CSD (90-95\% of cases): subacute regional lymphadenitis preceded by } \\
\text { cutaneous inoculation }\end{array}$ \\
$\begin{array}{ll}\text { 2. Systemic CSD (multiorgan involvement with rash) } \\
\text { ATYPICAL CSD }\end{array}$ \\
$\begin{array}{ll}\text { 3eliosis hepatis (dilated, blood cysts in the liver and spleen) } & \text { 1. CNS: seizure, headache, altered mental status, frequently encephalitis } \\
& \text { 2. Hepatosplenic: multiple low density lesions on abdominal imaging } \\
\text { 3. Cardiac: mitral and aortic valve vegetations multiple, rapidly progressive }\end{array}$ \\
$\begin{array}{ll}\text { 4. Parinaud's: conjunctivitis and regional preauricular lymphadenitis } \\
\text { 5. MSK: Myalgia, arthropathy, osteomyelitis, and tendinitis }\end{array}$
\end{tabular}

review, we have identified only 17 cases of HS-CSD in the past 10 years in adult patients.

Symptoms and Organ Involvement Visceral CSD can involve the liver, spleen, or both. Patients with visceral CSD may manifest fever of unknown origin (FUO), abdominalgia, weight loss, and hepatomegaly or splenomegaly on physical examination [19]. Many patients do not have peripheral adenopathy. In our case series, the most frequent clinical manifestation is fever in $100 \%$ of patients with or without headache and abdominal pain in $8 / 17$ patients $(47 \%)$. Liver and/or splenic involvement is often not associated with superficial lymphadenomegaly.

Immunodeficiency None of the observed patients had immunodeficiency. Indeed in literature, there are two distinct forms of hepatic involvement in CSD: the hepatic peliosis (seen in immunosuppressed patients, especially in HIV patient), which is an angioproliferative response to infection similar to bacillary angiomatosis and a HSCSD (seen in immunocompetent patients), in which the immune system provokes a granulomatous and/or suppurative response (Table 2) [20].

Laboratory Tests In literature the erythrocyte sedimentation rate (ESR) or C-reactive protein (CRP) are typically elevated, and liver function tests may be mildly abnormal. In our cases, a leukocytosis is present in $5 / 17$ patients $(29.4 \%)$, an elevation of CRP in $13 / 17$ patients $(76.4 \%)$, and alteration elevation of liver tests in $7 / 17$ patients $(41.2 \%)$ [9].

Diagnostic Tests In literature there is no definitive test for CSD. Combination of contact history and serological, clinical, and imaging criteria were considered compatible with HSCSD (Margileth diagnostic criteria).

To establish the diagnosis of CSD, literature suggested three of four of the following criteria [21]: [1] cat or flea contact with or without a lesion of inoculation; [2] negative serology for other causes of lymphadenopathy, sterile pus of a lymph node, positive
Bartonella PCR assay, and/or liver or spleen lesions on CT scan; [3] positive serology for B. henselae (titer $\geq 1: 64$ ); and [4] biopsy positive for granulomatous inflammation of lymph node or positive Warthin-Starry silver stain.

Imaging CT show multiple defects and abdomen ultrasound hypoechoic areas of the liver and/or in the spleen. US abdomen and abdomen CT are diagnostic imaging always used in the literature and in cases of our systematic review. In all cases reviewed, the lesions were studied with abdomen US and/or abdomen CT and/or abdomen MRI and/or PET scan. In our 2 cases, however, CEUS was of great help to the diagnosis and especially to the follow-up of patient, as it was a test more sensitive than ultrasound. CEUS can therefore help us to determine the duration of treatment.

Serology is the most used method, but in literature, its sensitivity is very variable $(<30-100 \%$, in indirect fluorescence assay:IFA and in enzyme immunosorbent assay:EIA) [22, 23]. Particularly specificities of IgG and IgM immunofluorescence for Bartonella henselae are 34-100\% and 86-100\% [24-27]. Both methods have some limitations: significant cross-reaction with B. Quintana (especially for IgG assays) and recent EBV infection (IgM), production of IgM brief, and therefore diagnosis of acute infection often difficult, false positive tests (prevalence of positive Bartonella serology in the general population is 4 to 6 percent $)^{1}$. In our case series and in systematic review, serology seems to have a good sensitivity: serology is positive in $14 / 17$ cases $(82.35 \%)$. In our second patient, serology was repeated after 5-7 days to obtain the diagnosis.

The sensitivity of the PCR is also not high: PCR sensitivity of sample tissue or aspirates is $30-60$ or $40-76 \%$, but specificity is high. The timing of the biopsy may influence the results: PCR seems to be more likely positive if it is performed during the first 6 weeks of infection [28]. In our second

\footnotetext{
${ }^{1}$ Low IgG may represent a past infection and not a current infection (if IFA IgG titers <1:64), IgG between 1:64 and 1:256 represent possible Bartonella infection (test should be repeated 10-14 days after), IgG $>1: 256$ suggest active or recent infection; positive IgM test suggests acute disease (or very recent infection).
} 
patient, PCR was required for the presence of new liver lesions, despite ongoing antibiotic therapy, but it was negative. In our systematic review (limitations are the small number of patients), the sensitivity of the CRP is low: $B$. henselae PCR was positive only in $7 / 17$ cases $(41 \%)$.

Bartonella grows very slowly, and it is hardly detectable in blood cultures. Culture is difficult even after 2-6 weeks of incubation: $B$. henselae is gram-negative, characterized by a very slow growth, and so it requires specific conditions: it is important to inform the laboratory of microbiology that Bartonella is a potential diagnosis (to optimize culture techniques and extend the incubation period for a minimum of 21 days). In our systematic review, the culture test was positive only in $2 / 17$ cases $(11.76 \%)$.

Histopathology shows granulomatous infection associated to acellular, necrotic center. $B$. henselae can be identified with Warthin-Starry silver stain ( $46 \%$ sensitive, rarely positive in the later stages). Histopathology of lymph node is often aspecific and variable with the stage of the disease: before lymphoid hyperplasia, then granulomas (centers acellular and necrotic; histiocytes and peripheral lymphocytes, similar to findings in skin samples), and at the end microabscesses confluent. Actually biopsies are rarely performed, and it should include histology, Warthin-Starry staining, PCR test, and tests to investigate other disorders. That is because clinical and imaging pictures are generally typical. A biopsy (lymph node or tissue) is indicated only in certain circumstances, as delayed resolution of systemic symptoms ( $>5-7$ days) and suspicion of other diagnosis.

In our case series and in our systematic review, a histopathological examination was obtained only in 9/17 patients $(52.9 \%)$, and it showed sometimes hepatic granulomas, sometimes hepatic abscesses, sometimes splenic granulomas, and sometimes granulomas or microgranulomas of lymph node.

Time for Clinical Resolution The mean to clinical remission is 4.9 weeks (range $2-12$ weeks), and the mean time to cure is 3.8 months (range 3-6 months).

Therapy Different therapy schemes were performed and with different duration (see Table 1 for details).

\section{Conclusions}

With the limitations of the low number of patients analyzed, the data would suggest the following:

- HS-CSD diagnosis is generally based on clinical features, imaging, and serologies, but sensitivity of serologies is very variable: negative serologic test not rule out diagnosis, if the clinical probability of patient is high. If there is a high clinical suspicion, the serology should be repeated.
- Histopathology is aspecific and shows granulomatous infection associated to acellular, necrotic center. $B$. henselae can be identified, but Warthin-Starry silver stain sensitivity (46\%, rarely positive in the later stages) and PCR sensitivity (40\%) are small. So a biopsy (lymph node or tissue) is indicated only in certain circumstances, as delayed resolution of systemic symptoms (>5-7 days) and suspicion of other diagnosis.

- In our case series, we have identified CEUS as a promising method for diagnosis and follow-up of HS-CSD.

US B mode accuracy for focal splenic lesions is $50 \%$ and Doppler US not add substantial help. The sensitivity of US is inferior to that of CT or MR and improves after the injection of US contrast agents: in literature CEUS sensitivity and specificity, positive and negative predictive values reached $100 \%, 83.8 \%, 87.8 \%$, and $100 \%$ for differentiating benign and malignant lesions [29] in liver and splenic nodules $[30,31]$. Indeed small ipoechoic/ isoechoic splenic or liver lesions may be difficult to identify with conventional US, but CEUS typically demonstrate no enhancement in any phase or rapid enhancement followed by persistent late-phase enhancement of benign splenic/liver lesions (Strong Consensus) [29]. Splenic abscesses typically appear as non-enhancing areas, or an enhanced border may be seen, especially in microabscesses. Instead malignant lesions demonstrate low early diffuse or peripheral enhancement followed by washout of microbubbles in the late phases. [32-34]. So evidences suggested that CEUS can be a helpful tool for health care professionals to confirm the diagnosis of solid lesions of liver and spleen [34].

However, the use of the CEUS in HS-CSD is not previously described in any of the cases of HS-CSD of the literature examined. Also in literature, there are few studies focused on the ability of CEUS to detect granulomatous lesions, such as in sarcoidosis. In observational studies, CEUS has the potential to become a valid and safe screening tool for systemic infiltration, as in sarcoidosis, and an important method of monitoring the effects of therapy [31, 35]. CT and MRI scans show granulomas as multiple, nonenhancing subcentimetric nodules, but these tools are associated with minor safety (contrast allergy, MRI-claustrophobia, pacemakers, metallic foreign bodies). CEUS allowed us to distinguish healthy (hyperechoic) liver and spleen parenchyma from small, avascular granulomas, and it is associated with increased security. Splenic and hepatic granulomas (or microabscess) are prevalent in HS CSD; instead we think that CEUS can be of great help in the diagnosis and the follow-up of these patients, even after an initial CT scan, because it is a sensitive method.

- A presumption diagnosis of HS-CSD generally involves empirical therapy. Within the limits of the few cases 
observed in the immunocompetent adult, a prolonged combination therapy may be beneficial in patients with severe disease or unresponsive to single therapy.

Combination therapy may reduce the possibility of $B$. henselae becoming sequestered in the bone marrow, lymph nodes, and thymus. For patients with disseminated disease or HS-CSD combination therapy (e.g., azithromycin plus rifampin or rifampin plus gentamicin or higher dose of azithromycin alone) can be prescribed. Rarely adjunctive corticosteroid therapy can also be administered.

- There are no specific markers for the follow-up. It may be reasonable to monitor the clinical response and C-reactive protein levels. Some believes that an imaging technique is required approximately 6 months after treatment, to document the lesion regression and others imaging tests are not necessary if the patient has complete clinical resolution.

In patients with HS-CSD, we believe that is it is useful to perform a follow-up with abdominal US and CEUS monthly; also after an initial CT scan, CEUS can be used for follow-up, to evaluate reduction in size of lesions, with no additional radiation exposure, as in patients with splenic trauma, abscesses, and infarcts [29].

- Several areas need to be explored by research: the role of CEUS in the diagnosis and follow-up of hepatosplenic abscesses and the correct modality of follow-up, antibiotic choice, combination treatment, treatment timing, and why some patients with HSHSD respond poorly to antibiotic therapy, even without an immunosuppression.

Acknowledgements The authors acknowledge Dr Michele Gaudio of Pathological Anatomy Institute, Morgagni Pierantoni Hospital, Forli'.

Code Availability Not applicable.

Author Contribution Each authors contributed equally to the writing of the article.

Data Availability Not applicable.

\section{Declarations}

Ethics Approval Not applicable.

Consent to Participate Not applicable.

Consent for Publication Patients gave written consent to the publication.

Conflict of Interest The authors declare no competing interests.

\section{References}

1. Bass JW, Vincent JM, Person DA. The expanding spectrum of Bartonella infections: II. Cat-scratch disease. Pediatr Infect Dis J. 1997;16:163.

2. Spach DH, Koehler JE. Bartonella-associated infections. Infect Dis Clin North Am. 1998;12:137.

3. Jacomo V, Kelly PJ, Raoult D. Natural history of Bartonella infections (an exception to Koch's postulate). Clin Diagn Lab Immunol. 2002;9:8.

4. Dehio C. Molecular and cellular basis of Bartonella pathogenesis. Annu Rev Microbiol. 2004;58:365.

5. Verma SK, Martin A, Montero J. Atypical cat scratch disease with hepatosplenic involvement. Clin Gastroenterol Hepatol. 2017 Jan;15(1):e5-6.

6. Knafl D, Lötsch F, Burgmann H. Hepatosplenic abscesses and osteomyelitis of the spine in an immunocompetent adult with cat scratch disease. Case Rep Infect Dis. 2015;2015:317260.

7. Bieraugel K, Oehler D, NeSmith M. Cat got your spleen? Hepatosplenic Bartonella infection Am J Med. 2015 Mar;128(3): 246-9.

8. Shasha D, Gilon D, Vernea F. Visceral cat scratch disease with endocarditis in an immunocompetent adult: a case report and review of the literature. VECTOR-BORNE AND ZOONOTIC DISEASES. 2014;14(3):p175-18.

9. García JC, MD NMJ, Castro B, et al. Hepatosplenic cat scratch disease in immunocompetent adults. Report of 3 Cases and Review of the Literature. Medicine (Baltimore). 2014 Oct;93(17): 267-79.

10. Anyfantakis D, Kastanakis M, Papadomichelakis A, et al. Catscratch disease presenting as a solitary splenic abscess in an immunocompetent adult: case report and literature review. Le Infezioni in Medicina, n. 2013;2:130-3.

11. Liberto MC, Matera G, Lamberti AG, et al. Diagnosis and followup of Bartonella henselae infection in the spleen of an immunocompetent patient by real-time quantitative PCR. J Med Microbiol. 2013;62:1081-5.

12. VanderHeyden TR, Yong SL, Breitschwerdt EB, et al. Granulomatous hepatitis due to Bartonella henselae infection in an immunocompetent patient. BMC Infect Dis. 2012;12:17.

13. Graveleau J, Grossi O, Lefebvre M, et al. Vertebral osteomyelitis: an unusual presentation of Bartonella henselae infection. Semin. Arthritis Rheum. 2011;41:511-6.

14. Zenone T. Systemic Bartonella henselae infection in immunocompetent adults presenting as fever of unknown origin. Case Report Med. 2011;2011:183397.

15. Ben-Ami R, Ephros M, Avidor B, et al. Cat-scratch disease in elderly patients. Clin Infect Dis. 2005;41:969.

16. Lenoir AA, Storch GA, DeSchryver-Kecskemeti K, et al. Granulomatous hepatitis associated with cat scratch disease. Lancet. 1988;1:1132.

17. Delahoussaye PM, Osborne BM. Cat-scratch disease presenting as abdominal visceral granulomas. J Infect Dis. 1990;161:71.

18. Fretzayas A, Papadopoulos NG, Moustaki M, et al. Unsuspected extralymphocutaneous dissemination in febrile cat scratch disease. Scand J Infect Dis. 2001;33:599.

19. Arisoy ES, Correa AG, Wagner ML, Kaplan SL. Hepatosplenic catscratch disease in children: selected clinical features and treatment. Clin Infect Dis. 1999;28:778.

20. Tappero JW, Koehler JE, Berger TG, et al. Bacillary angiomatosis and bacillary splenitis in immunocompetent adults. Ann Intern Med. 1993;118:363.

21. Margileth AM. Recent advances in diagnosis and treatment of cat scratch disease. Current Infectious Disease Reports. 2000;2(2): $141-6$. 
22. English CK, Wear DJ, Margileth AM, et al. Cat-scratch disease. Isolation and culture of the bacterial agent. JAMA. 1988;259:1347.

23. Brenner DJ, Hollis DG, Moss CW, et al. Proposal of Afipia gen. nov., with Afipia felis sp. nov. (formerly the cat scratch disease bacillus), Afipia clevelandensis sp. nov. (formerly the Cleveland Clinic Foundation strain), Afipia broomeae sp. nov., and three unnamed genospecies. J Clin Microbiol. 1991;29:2450.

24. Bergmans AM, Peeters MF, Schellekens JF, et al. Pitfalls and fallacies of cat scratch disease serology: evaluation of Bartonella henselae-based indirect fluorescence assay and enzyme-linked immunoassay. J Clin Microbiol. 1997;35:1931.

25. Dupon M, Savin De Larclause AM, Brouqui P, et al. Evaluation of serological response to Bartonella henselae, Bartonella quintana and Afipia felis antigens in 64 patients with suspected cat-scratch disease. Scand J Infect Dis. 1996;28:361.

26. Sander A, Posselt M, Oberle K, Bredt W. Seroprevalence of antibodies to Bartonella henselae in patients with cat scratch disease and in healthy controls: evaluation and comparison of two commercial serological tests. Clin Diagn Lab Immunol. 1998;5:486.

27. Zbinden R, Michael N, Sekulovski M, et al. Evaluation of commercial slides for detection of immunoglobulin $G$ against Bartonella henselae by indirect immunofluorescence. Eur J Clin Microbiol Infect Dis. 1997;16:648.

28. Ridder GJ, Boedeker CC, Technau-Ihling K, et al. Role of catscratch disease in lymphadenopathy in the head and neck. Clin Infect Dis. 2002;35:643.
29. Hopkins A, Cokkinos DD. Contrast enhanced sonographic study of the spleen. HJR. 2(1):49-65.

30. Berzigotti A, Ferraioli G, Bota S, Gilja OH, Dietrich CF. Novel ultrasound-based methods to assess liver disease: the game has just begun. Dig Liver Dis. 2018;50:107-12.

31. Tana C, Schiavone C, Ticinesi A, Ricci F, Giamberardino MA, Cipollone F, et al. Ultrasound imaging of abdominal sarcoidosis: state of the art. World J Clin Cases. 2019;7(7):809-18.

32. Omar A, Freeman S. Contrast-enhanced ultrasound of the spleen. Ultrasound. 2016;24(1):41-9.

33. GORG C. The forgotten organ: contrast enhanced sonography of the spleen. Eur. J. Radiol. 2007;64(2):189-201.

34. Sidhu PS, Cantisani V, Dietrich CF, Gilja OH, Saftoiu A, Bartels E, et al. The EFSUMB guidelines and recommendations for the clinical practice of contrast-enhanced ultrasound (CEUS) in nonhepatic applications: update 2017 (long version). Ultraschall Med. 2018;39:e2-e44.

35. Grzelak P, Augsburg L, Majos A, Stefańczyk L, Górski P, Piotrowski W, et al. Diagnostic potential of contrast-enhanced ultrasound (CEUS) in the assessment of spleen and liver granulomas in the course of sarcoidosis. Pneumonol Alergol Pol. 2013;81(5): $424-8$.

Publisher's Note Springer Nature remains neutral with regard to jurisdictional claims in published maps and institutional affiliations. 